Howard B. Beckwith*

Nagoya Math. J.

Vol. 40 (1970), 121-131

\title{
ON TRACE FOR MODULES
}

\author{
HOWARD B. BECKWITH*
}

\section{Introduction}

Classically, trace was defined as the sum of the diagonal entries of a square matrix with entries in a field. This notion played an important role in classical mathematics, e.g. in the theory of algebras over a field of characteristic zero, and in the theory of group characters (as in [1]). A generalization to endomorphisms of a finitely generated projective module over any ring $R$ with unit is well-known. For such a module $P$ the canonical homomorphism $\Psi: P^{*} \otimes P \rightarrow \operatorname{End}_{R}(P)$ is an isomorphism. Then the composite $\varepsilon \circ \Psi^{-1}: \operatorname{End}_{R}(P) \rightarrow P^{*} \otimes P \rightarrow R$, where $\varepsilon$ denotes "evaluation", is a homomorphism which coincides with the classical trace whenever $P$ is free. This version of trace has been used by Hattori [3] and others to study projective modules. However, this approach to trace is limited to the finitely generated projective modules, since it can be shown that $\Psi$ is an isomorphism if and only if $P$ is finitely generated and projective.

In [5] an approach is supplied to get beyond the projective case. There trace is taken to be an $R$-module homomorphism $t: \operatorname{End}_{R} M \rightarrow T$ which is "universal" in the category of $R$-modules with respect to the basic trace property: $t(\mu)=t\left(\alpha \mu \alpha^{-1}\right)$ for all $\mu \in \operatorname{End}_{R} M$ and $\alpha \in \mathrm{Aut}_{R} M$. Existence is shown not only for modules, but also for a much broader categorical context. In [5] this trace is studied in additive categories and in the category of $R$-modules, including a comparison with classical definitions. In this paper we continue the study, treating trace (for modules) with respect to the constructions: (1) localization at a prime ideal, (2) forming quotients modulo an ideal and (3) completions. This leads to some techniques of reducing certain question from a general setting, over an arbitrary commutative ring, to the level of modules over a local ring, a complete ring,

Received May 27, 1969.

* Research partially sponsored by the Air Force Office of Scientific Research, Office of Aerospace Research, United States Air Force, under AFOSR Grant AF-AFOSR-920-65. 
or even a field. We then consider the question when an nilpotent endomorphism has trace equal to zero (see §2). Finally, we give an alternate version of Hattori's rank element theorem.

This is a revision of a portion of the author's dissertation. The author is very much indebted to Helmut Röhrl for suggesting the problem and for his guidance.

\section{$\S 1$. Definitions and Preliminaries}

We collect together for reference the definitions and some basic facts, essentially from [5], in the form in which they are needed in this paper. Throughout, $R$ denotes a commutative ring with unit; $\operatorname{Mod}(R)$ denotes the category of $R$-modules.

(1.1) Definition ([5], $\$ 1$ and $\S 3$ ). By trace for an $R$-module $M$ we mean the unique (up to isomorphism) solution in $\operatorname{Mod}(R)$ of the following universal problem: an $R$-homomorphism $t: \operatorname{End}_{R}(M) \rightarrow T$ satisfying (i) $t(\mu)=t\left(\alpha \mu \alpha^{-1}\right)$ for all $\mu \in \operatorname{End}_{R}(M)$ and all $\alpha \in \operatorname{Aut}_{R}(M)$, and (ii) for any $R$-homomorphism $t^{\prime}: \operatorname{End}_{R}(M) \rightarrow T^{\prime}$ satisfying condition (i) there is a unique $R$-homomorphism $\tau: T \rightarrow T^{\prime}$ such that $t^{\prime}=\tau t$, i.e., so that the following diagram commutes:

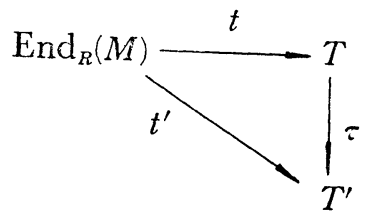

Condition (ii) is referred to as the universality of trace. The following construction of trace for $M$ is important for us here: let $S$ be the $R$ submodule of $\operatorname{End}_{R}(M)$ generated by all elements of the form $\mu-\alpha \mu \alpha^{-1}$ with $\mu \in \operatorname{End}_{R}(M)$ and $\alpha \in \operatorname{Aut}_{R}(M)$, then trace for $M$ is precisely the canonical epimorphism, denoted $\operatorname{tr}_{M}: \operatorname{End}_{R}(M) \rightarrow \operatorname{End}_{R}(M) / S$. Thus, $\operatorname{Tr}_{M}=\operatorname{End}_{R}(M) / S$.

Now, it turns out that in practice, in order to compute with various constructions we require the following commutative rule, which is clearly a strengthening of condition (i) of (1.1):

$$
t(\mu \nu)=t(\nu \mu) \text { for all } \mu, \nu \in \operatorname{End}_{R} M .
$$

Since this may not hold in general for trace as given above, we turn naturally to the "commutative companion" as in [5]: 
(1.2) Definition. By commutative trace for an $R$-module $M$ we mean the unique solution of the universal problem in (1.1) but with condition (i) replaced with condition (i)'.

This trace is denoted $\operatorname{tr}_{M}^{c}: \operatorname{End}_{R}(M) \rightarrow \operatorname{Tr}_{M}^{c}$. It is easily seen to be the canonical epimorphism $\operatorname{End}_{R}(M) \rightarrow \operatorname{End}_{R}(M) / S^{c}$ where $S^{c}$ is the $R$-submodule of $\operatorname{End}_{R}(M)$ generated by all elements $\mu \nu-\nu \mu$ with $\mu, \nu \in \operatorname{End}_{R}(M)$. There is an obvious epimorphism $\operatorname{Tr}_{M} \rightarrow \operatorname{Tr}_{M}^{c}$; if this is an isomorphism, we say that trace is commutative (for $M$ ). We now quote from [5]:

(1.3) Proposition. If $\mu \in \operatorname{End}_{R}(M)$ is in the R-submodule of $\operatorname{End}_{R} M$ that is generated by $A u t_{R}(M)$, then for every $\nu \in \operatorname{End}_{R}(M) \operatorname{tr}_{M}(\mu \nu)=\operatorname{tr}_{M}(\nu \mu)$.

This result suggests the following definition

(1.4) Definition. An $R$-module $M$ has the Aut-End property if the set $\operatorname{Aut}_{R}(M)$ is a set of generators of the $R$-module $\operatorname{End}_{R}(M)$.

(1.5) Proposition. (cf. [5] (3.3), (3.4)) For any R-module $M$

(i) If $M$ has the Aut-End property, then trace is commutative for $M$.

(ii) If $M$ is a finite direct sum of at least two copies of some $R$-module, then $M$ has the Aut-End property.

Most of what follows deals with commutative trace. Now, trace cannot be expected to be functorial, but the following is a useful replacement (cf. [5] (1.11)).

(1.6) Proposition. Let $R_{1}$ and $R_{2}$ be commutative rings with units and $\rho: R_{1} \rightarrow R_{2}$ a unital ring homomorphism. Let $\rho_{*}: \operatorname{Mod}\left(R_{2}\right) \rightarrow \operatorname{Mod}\left(R_{1}\right)$ denote "restriction of scalars". If $M_{i} \in \operatorname{Mod}\left(R_{i}\right)$ for $i=1,2$, and $k: \operatorname{End}_{R_{1}}\left(M_{1}\right) \rightarrow \rho_{*} \operatorname{End}_{R_{2}}\left(M_{2}\right)$ is an $R$-monoid homomorphism, then there exist unique $R_{1}$-homomorphisms $\bar{k}^{c}$ and $r^{c}(\rho)$ such that the following diagram commutes:

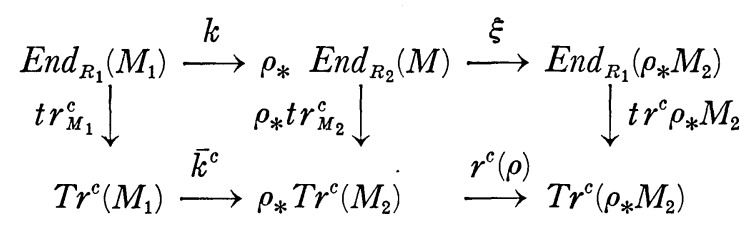

where $\xi$ denotes the canonical $R_{1}$-homomorphism. If $k$ is surjective, then so is $\bar{k}^{c}$. If $\rho$ is an epimorphism, then $r^{c}(\rho)$ is an isomorphism.

Proof. See [5], (1.11) and (4.7). The analogous result holds for trace. 
The first instance of this Proposition is the construction of modules of quotients relative to a multiplicative subset $S \subseteq R$ (assume $0 \notin S, 1 \in S$ ). If $M$ is any $R$-module, then the functor $S^{-}: \operatorname{Mod}(R) \rightarrow \operatorname{Mod}\left(S^{-1} R\right)$ induces a map $k$ as in (1.6), and so leads to a unique $R$-homomorphism $\sigma_{S}^{c}$ such that the following diagram of $R$-modules commutes:

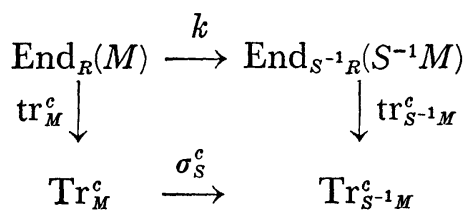

(1.7) Proposition. In the preceding notation, if the canonical $S^{-1} R$-homomorphism $S^{-1} E_{n} M \rightarrow \operatorname{End}_{S^{-1} R}\left(S^{-1} M\right)$ is an isomorphism (e.g., when $M$ is finitely presentable), then

(i) If $t r_{M}$ is commutative, then so is $t r_{S^{-1} M}$

(ii) If $M$ has the Aut-End property, then so does $S^{-1} M$ as an $S^{-1} R$-module.

(iii) $\operatorname{ker} \sigma_{S}^{c}=\left\{t \in \operatorname{Tr}_{M}^{c}: \operatorname{ann}(t) \cap S \neq \phi\right\}$

Proof: In the spirit of [5] (4.9).

(1.8) Corollary. Let $M$ be an R-module such that for each maximal ideal $m$ of $R$, the canonical $\left[\operatorname{End}_{R}(M)\right]_{m} \rightarrow \operatorname{End}_{R_{m}}\left(M_{m}\right)$ is an $R_{m}$-isomorphism where ()$_{m}$ denotes $(R-m)^{-1}(\quad)$. Denote $\lambda^{c}(m)=\sigma_{R-m}^{c}$. Then $\cap\left\{k e r \lambda^{c}(m): m\right.$ is a maximal ideal in $R\}=(0)$.

We turn now to a second instance of (1.6). If $A$ is an ideal in $R$, the assignment $M \rightarrow M / A M$ prescribes a right exact, covariant additive functor $Q_{A}: \operatorname{Mod}(R) \rightarrow \operatorname{Mod}(R / A) . \quad\left(Q_{A}\right.$ is of course naturally equivalent to $(R / A)$ $\otimes_{R}$-.) For any $R$-module $M, Q_{A}$ induces a map $k=Q_{A} \mid \operatorname{End}_{R}(M)$ as in (1.6) and then a unique $R$-homomorphism $\phi^{c}$ such that the following diagram commutes:

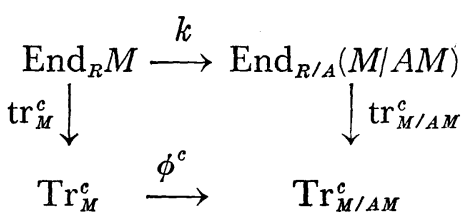

In order to treat the kernel of $\phi^{c}$ we consider first the $R / A$-homomorphism $\eta: Q_{A} \operatorname{Hom}_{R}(M, N) \rightarrow \operatorname{Hom}_{R / A}\left(Q_{A} M, Q_{A} N\right)$ induced by $Q_{A}$. Our primary interest 
lies in the question when $\eta$ is surjective. We now give various instances of this.
(1.10) Proposition.
(i) $\operatorname{ker} \eta=\operatorname{Hom}_{R}(M, A N) / A \cdot \operatorname{Hom}_{R}(M, N)$
(ii) If $M$ is R-projective, then $\eta$ is surjective for any $N$.
(iii) If $A$ is finitely generated and $M$ is projective, then $\eta$ is bijective.
(iv) If $A$ is finitely generated, then $\eta$ is surjective, for all $M$ and $N$.

Proof. (i) and (ii) are easily checked. So assume $A$ is finitely generated and $M$ is projective. Due to (ii) we need only show $\eta$ is injective. Due to (i) this follows if $\operatorname{Hom}_{R}(M, A N) \leqq A \cdot \operatorname{Hom}(M, N)$. For this, let $f \in \mathrm{Hom}_{R}$ $(M, A N)$ and suppose $A$ is generated by the elements $a_{1}, \cdots, a_{n} \in R$. These elements induce the $R$-homomorphism $r: N \oplus \cdots \oplus N(n$-fold $) \rightarrow A N$ by $\gamma\left(x_{1}, \cdots, x_{n}\right)=\sum_{i=1}^{n} a_{i} x_{i}$. Since the $a_{i}$ generate $A, \gamma$ is surjective. Projectivity of $M$ then allows to factor:

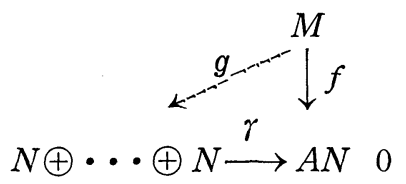

Now if $\pi_{i}$ is the $i^{t h}$ projection: $N \oplus \cdots \oplus N \rightarrow N$ and $f_{i}=\pi_{i} g$, then $f=\sum_{i=1}^{n} a_{i} f_{i}$ is easily computed. This proves (iii). (iv). Let $0 \rightarrow K \stackrel{k}{\rightarrow} P \stackrel{\pi}{\rightarrow} M \rightarrow 0$ be an exact sequence of $R$-modules with $P$ projective. Then setting $H=\mathrm{Hom}_{R}$ $(-, N)$, we have an exact sequence $H(M) \stackrel{\pi^{*}}{\rightarrow} H(P) \stackrel{k^{*}}{\rightarrow} H(K)$. Let $I=i m\left(k^{*}\right)$ to derive the exact sequence $H(M) \rightarrow H(P) \rightarrow I \rightarrow 0$. Apply $Q=Q_{A}$ to see finally $Q H(M) \rightarrow Q H(P) \rightarrow Q I \rightarrow 0$ is exact. Similarly, if $\bar{H}=\mathrm{Hom}_{R / A}(-, N / A N)$, we have the exact sequence along the bottom of the following commutative diagram, with $x$ induced by $Q$ :

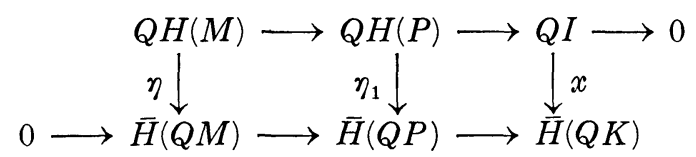

Due to (iii), $A$ finitely generated, and $P$ projective $\eta_{1}$ is bijective. Suppose. $x(f+A I)=0$. Since $f \in I$, there exists $g \in \operatorname{Hom}(P, N)$ such that $f=g \pi$. We 
may view $g \in \operatorname{Hom}(P, A N)$, since $x(f+A I)=0$. But $P$ projective, $A$ finitely generated and the proof of (iii) show that $\operatorname{Hom}(P, A N)=A \operatorname{Hom}(P, N)$. Writing $g=\sum a_{i} g_{i}$ with $g_{i} \in \operatorname{Hom}(P, N)$, we see that $f=\sum a_{i}\left(g_{i} \pi\right) \in A I$. This shows that $x$ is injective. It now follows by routine diagram chasing that $\eta$ is surjective. This completes the proof of (1.10).

Now we are ready to study (1.9) further. Note that the map $k$ of (1.9) is surjective if and only if the map $\eta$ of (1.10) is surjective.

(1.12) Proposition. If $k$ (of (1.9)) is surjective, then

$$
\operatorname{ker} \phi^{c}=\operatorname{tr}_{M}^{c}(k e r k)
$$

Proof. Let $\tau=\operatorname{tr}_{M}^{c}(\mu) \in \operatorname{ker} \phi^{c}$. By commutativity of (1.9) and surjectivity of $k$ we may write

$$
k(\mu)=\sum_{i}\left[k\left(x_{i}\right) k\left(y_{i}\right)-k\left(y_{i}\right) k\left(x_{i}\right)\right]
$$

for suitable $x_{i}, y_{i} \in \operatorname{End}_{R}(M)$. It follows $\mu-\sum\left(x_{i} y_{i}-y_{i} x_{i}\right) \in \operatorname{ker}(k)$. Then $\mu \in \operatorname{ker}(k)+\operatorname{ker} \operatorname{tr}_{M}^{c}$, and finally $\tau=\operatorname{tr}_{M}^{c}(\mu) \in \operatorname{tr}_{M}^{c}(\operatorname{ker}(k))$. The converse is trivial.

(1.13) Proposition. For any $R$-module $M$ and ideal $A$ in $R, A \operatorname{Tr}_{M}^{c} \leqq k e r \phi^{c}$. If in addition $k$ is surjective, then we have $A \cdot \operatorname{Tr}_{M}^{c}=k e r \phi^{c}$ if and only if $\operatorname{Hom}(M, A M) \leqq A \cdot \operatorname{Hom}(M, M)+\operatorname{ker}\left(\operatorname{tr}_{M}^{c}\right)$.

Proof. The first assertion follows from the commutativity of (1.9). For the second assertion, assume $\operatorname{ker} \phi^{c} \subseteq A \cdot \operatorname{Tr}_{M}^{c}$ and $\mu \in \operatorname{Hom}(M, A N)=\operatorname{ker}(k)$. Then $\operatorname{tr}_{M}(\mu) \in \operatorname{tr}_{M}(\operatorname{ker} k)=\operatorname{ker} \phi^{c}=A \cdot \operatorname{Tr}_{M}^{c}$. From this it follows quickly $\mu \in A$. End $(M)+\operatorname{ker} \operatorname{tr}_{M}^{c}$, which completes the proof.

We present finally the following statement concerning the Hom condition of the preceding proposition. Note that the Ext-condition is satisfied automatically when $M$ is projective.

(1.14) Proposition. Let $M$ and $N$ be $R$-modules, $A$ a finitely generated ideal in R. If $A$ is generated by $n$ elements and if for every submodule $K$ of $N^{n}=N \oplus \cdots \oplus N$ $(n-f o l d) \operatorname{Ext}(M, K)=0$ holds, then $\operatorname{Hom}_{R}(M, A N)=A \cdot H_{0}(M N)$.

Proof. The proof follows the argument used in proving (ii) of (1.10). We need only the existence of $g$ in (1.11), but this follows from the exact sequence 


$$
\operatorname{Hom}_{R}\left(M, N^{n}\right) \rightarrow \operatorname{Hom}_{R}(M, A N) \rightarrow \operatorname{Ext}(M, K)=0
$$

where $K=\operatorname{ker}(\gamma)$.

\section{§2. Trace and Nilpotent Endomorphisms}

In this section we turn to various results concerning the question when nilpotent endomorphisms have zero trace. It is good to point out that this cannot always be the case, as the following simple example shows. Suppose $R$ is a ring and $r \neq 0$ is a nilpotent element of $R$, and $I_{n}$ is the identity matrix of order $n$ over $R$, then the matrix $r \cdot I_{n}$ is nilpotent, but $\operatorname{tr}\left(r \cdot I_{n}\right)=n \cdot r$ need not be zero. Thus some restriction is necessary. The type of restriction which arises in this section concerns the Jacobson radical, $J(M)$, of the $R$-module $M$, defined to be the intersection of the maximal submodules of $M$, or to be equal to $M$ when $M$ has no maximal submodules. It is known that when $M$ is noetherian, then $J(M)$ is the intersection of the submodules $m M$ as $m$ ranges through the maximal ideals of $R$. Also $J(M)=(0)$ if and only if $M$ can be imbedded in a direct product of simple $R$-modules. Our first statement is the most general we are able to derive from the construction (1.9).

(2.1) Proposition. If $M$ is a noetherian $R$-module such that $k$ is surjective and $\operatorname{Hom}_{R}(M, A M) \subseteq A \operatorname{Hom}_{R}(M, M)$ for all maximal ideals $A$ in $R$, and if $J\left(\operatorname{Tr}_{M}^{c}\right)=(0)$, then $\operatorname{tr}_{M}^{c}(\mu)=0$ for every nilpotent $\mu \in \operatorname{End}_{R}(M)$.

Proof. By (1.13), the commutativity of (1.9) and the fact that $\mu$ nilpotent implies $k^{c}(\mu)$ is also nilpotent, the classical result for trace over fields leads to $\operatorname{tr}_{M}^{c}(\mu) \in \cap\left\{A \cdot \operatorname{Tr}_{M}^{c}: A\right.$ is a maximal ideal in $\left.R\right\}=J\left(\operatorname{Tr}_{M}\right)$. Since $J\left(\operatorname{Tr}_{M}\right)=0$, this completes the proof.

Of course this includes the result that trace of a nilpotent endomorphism is zero when $M$ is a finitely generated projective module over a (commutative) ring $R$ such that $J(R)=(0)$. (cf. the example above).

For other results of this type we consider local and semi-local rings.

(2.2) Proposition. If $R$ is noetherian, $M$ a finitely generated $R$-module with $J\left(M_{A}\right)=0$ for each maximal ideal $A$ in $R$, then $\operatorname{tr}_{M}^{c}(\mu)=0$ for each nilpotent $\mu \in \operatorname{End}_{R}(M)$.

Proof. Since $\mu$ nilpotent implies $k(\mu)$ is nilpotent, referring to the diagram preceding (1.7), (1.8) reduces our considerations to the case that $R$ is a local 
noetherian ring. In that case the unique maximal ideal $A$ is finitely generated, and thus by (1.10) $k$ of (1.9) is surjective. Also $J(M)=A \cdot M=(0)$, since $M_{A} \cong M$ and this implies $J\left(\operatorname{Tr}_{M}^{c}\right)=A \cdot \operatorname{Tr}_{M}^{c}=0$. Thus (2.1) applies to complete the proof.

(2.3) Corollary. If $R$ is a noetherian semilocal ring and $M$ a finitely generated $R$-module such that $J(M)=(0)$, then $\operatorname{tr}_{M}^{c}(\mu)=0$ for all nilpotent $\mu \in \operatorname{End}_{R}(M)$.

Proof. We show that $R$ being semilocal implies that for each maximal ideal $A J\left(M_{A}\right)=J\left(M_{A}\right)$. To see this, let $x=p m / s \in J\left(M_{A}\right)=A \cdot M_{A}$ with $p \in A, m \in M$, $s \notin A$. Let $A=A_{1}, A_{2}, \cdots, A_{g}$ be the maximal ideals in $R$ and let $p_{i} \in A_{i}-A_{1}$ for each $i>1$. Putting $n=\pi\left\{p_{i}: i>1\right\}$ we have $n p m \in \cap A_{i} M=J(M)$ but also $n s \notin A$, which shows $x=p m / s=n p m / n s \in(J M)_{A}$. The converse is trivial. From this formula we deduce that $J(M)=0$ implies $J\left(M_{A}\right)=0$ for each maximal ideal, and (2.2) completes the proof.

Finally since it is known that if $R / J R$ is semisimple, then $J(M)=J(R) \cdot M$, we also have:

(2.4) Corollary. Let $R$ be a noetherian semilocal ring such that $R / J R$ is semisimple. if $M$ is a finitely generated $R$-module. such that $J(R) \cdot M=0$, then $\operatorname{tr}_{M}^{c}(\mu)=0$ for each nilpotent $\mu \in \operatorname{End}_{R}(M)$.

\section{§3. Trace and Completions}

In this section we utilize the results in [2] on linear topologies. See also Nagata [4], and Zariski-Samuel [6]. Throughout $R$ denotes a commutative noetherian ring, and $A$ an ideal in $R$ such that $A \subseteq J(R)$. By the $A$-preadic topology for an $R$-module $M$ is meant the linear topology gotten by using the powers $A^{n} M, n=1,2,3, \cdots$ as a fundamental system of open neighborhoods of 0 . The assumption $A \subseteq J(R)$ assures that every finitely generated $R$-module is hausdorff, and each of its submodules is closed, and its $A$-preadic topology coincides with the induced topology. We denote here by $C M$ the completion of $M$ for the $A$-preadic topology, namely $C M=$ proj. $\lim . M / A^{n} M$. In [2] it is shown that under our hypotheses $C$ is an exact functor on the category of finitely generated $R$-modules, and for every pair of finitely $R$-modules, $M$ and $N: C \cdot \operatorname{Hom}_{R}(M, N) \cong \operatorname{Hom}_{C R}(C M, C N)$ and the latter is complete.

Now given a $R$-module $M$ there is a commutative diagram 


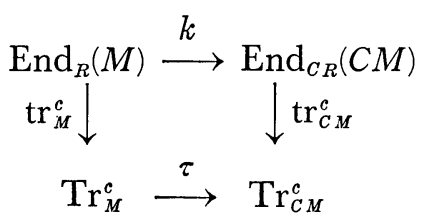

due to (1.6) with $k$ induced by the functor $C$.

(3.2) Proposition. If $M$ is finitely generated, then $\tau$ is a monomorphism and $\operatorname{Tr}_{C M}^{c} \cong C \operatorname{Tr}_{M}^{c}$ such that the following commutes:

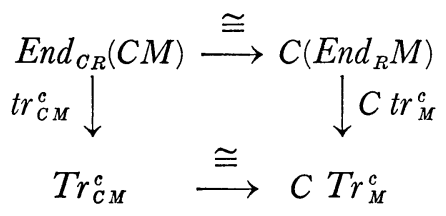

Proof. $\operatorname{Tr}_{C M}^{c} \rightarrow C \operatorname{Tr}_{M}^{c}$ is induced by universality of trace since the top isomorphism is a ring isomorphism. To construct the inverse, note $\operatorname{End}_{C R}(C M)$ complete implies $\operatorname{Tr}_{C M}^{c}$ is also complete, since $\operatorname{tr}_{C M}^{c}$ is continuous and Cauchy sequences have Cauchy preimages.

\section{§4. The Rank Element}

In [3] Hattori defines the rank element, $r_{A}(P)$, of a finitely generated projective $A$-module $P$ to be the trace of the identity endo-morphism of $P$, where he took trace to be the composite

$$
\operatorname{End}_{A}(P) \stackrel{\theta^{-1}}{\longrightarrow} P^{*} \stackrel{\pi}{\longrightarrow} A^{a}
$$

Here, $\theta: P^{*} \otimes P \rightarrow \operatorname{End}_{A}(P)$ is the canonical isomorphism, and $\pi$ denotes evaluation $P^{*} \otimes P \rightarrow A$ followed by the canonical quotient map $A \rightarrow A /[A, A]$, $[A, A]$ the being ideal generated by the set of all elements $a b-b a$ with $a, b \in A$. He proves

Theorem (Hattori). Let $A$ be an algebra over a complete local ring $\sigma$, finitely generated as an $\sigma$-module. If $\sigma / m$ has characteristic $p \neq 0$, where $m=J(\sigma)$, we then assume $A^{a}$ is $p$-torsion free. Then two finitely generated projective $A$-modules, $P_{1}$ and $P_{2}$, are isomorphic if and only if $r_{A}\left(P_{1}\right)=r_{A}\left(P_{2}\right)$.

We now give an alternate version of this theorem, in the case the algebra is commutative. The restriction to commutative algebras arises only 
in order to have (4.11) in [5]. As in Hattori we define $r_{A}(M)=\operatorname{tr}_{M}\left(1_{M}\right)$ for any $A$-module $M$.

(4.1) Theorem. Let $A$ be a commutative algebra over a noetherian local ring $\sigma$, finitely generated as an $\sigma$-module. If the characteristic of $\sigma / m$ is $p \neq 0$, we assume further that $C A$ has no p-torsion. Let $P_{1}$ and $P_{2}$ be finitely generated projective $A$ modules with commutative trace, and suppose some power of $m$ annihilates either $P_{1}$ or $P_{2}$. Then $P_{1}$ and $P_{2}$ are isomorphic if and only if $r_{A}\left(P_{1}\right)=r_{A}\left(P_{2}\right)$.

Proof. We begin by noting that if $m=J(\sigma)$ is nilpotent (e.g. if $\sigma$ is artinian), the annihilator condition is automatically satisfied. Completions are relative to the ideal $m A$. We may view $\operatorname{Tr}_{P_{i}} \subseteq A, i=1,2$ and form the commutative diagram:

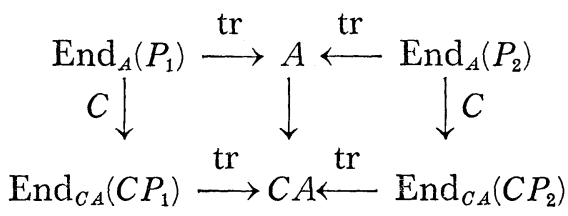

where $A \rightarrow C A$ is the canonical insertion, due to [5] (4.11) and [2] (0.7.3.4). Each corresponds to (3.4). Since $C\left(1_{P_{1}}\right)=1_{C P_{1}}$ and $C\left(1_{P_{2}}\right)=1_{C P_{2}}$, it follows $r_{A}\left(P_{1}\right)=r_{A}\left(P_{2}\right)$ implies $r_{C A}\left(C P_{1}\right)=r_{C A}\left(C P_{2}\right)$. Since $C A$ is an algebra over the complete local ring $C_{m}(\sigma)$, our hypotheses allow to apply the Hattori theorem, to deduce $C P_{1} \cong C P_{2}$. Let $\phi: C P_{1} \rightarrow C P_{2}$ be any isomorphism. Then $\phi, \phi^{-1} \in \mathrm{Hom}_{C A}\left(C P_{1}, C P_{2}\right)$. Since $\operatorname{Hom}_{C A}\left(C P_{1}, C P_{2} \cong C \operatorname{Hom}_{A}\left(P_{1}, P_{2}\right)\right.$, we may identify $\phi=\left\{\phi_{n}\right\}$ and $\phi^{\prime}=\left\{\phi n^{\prime}\right\}$, with $\phi_{n}, \phi_{n}^{\prime} \in \operatorname{Hom}_{A}\left(P_{1}, P_{2}\right)$ for each $n$. Since $\phi \phi^{\prime}=1$, we may conclude that $\phi_{n} \phi_{n}^{\prime}-1 \in m^{n} \operatorname{Hom}_{A}\left(P_{1}, P_{2}\right)$. But for sufficiently large $n$ this is zero, and for such $n, \phi_{n} \phi_{n}^{\prime}=1$. Similarly $\phi_{n}^{\prime} \phi_{n}=1$ for large $n$. Thus $\phi_{n}: P_{1} \rightarrow P_{2}$ is an isomorphism for large $n$. This completes the proof of (4.1), since the converse is clear.

Following Hattori it is now possible to deduce

(4.2) Corollary. Let $\sigma$ be a (noetherian) local domain of characteristic zero with quotient field $K$ and let $A$ be a commutaive $\sigma$-algebra with no $\sigma$-torsion and finitely generated as an o-module. Assume $P_{1}$ and $P_{2}$ are finitely generated projective A-modules with commutative trace and with some power of $m=J(\sigma)$ annihilating $P_{2}$ (or $P_{1}$ ). Then $P_{1} \cong P_{2}$ if and only if $K \otimes_{\sigma} P \cong K \otimes_{\sigma} P_{2}$.

Proof. Since $P_{1}$ and $P_{2}$ are finitely generated and projective, as are $K \otimes P_{1}$ 
and $K \otimes P_{2}$, we may identify $\operatorname{Tr}_{P_{2}} \cong A$ as before and get the follwing diagram:

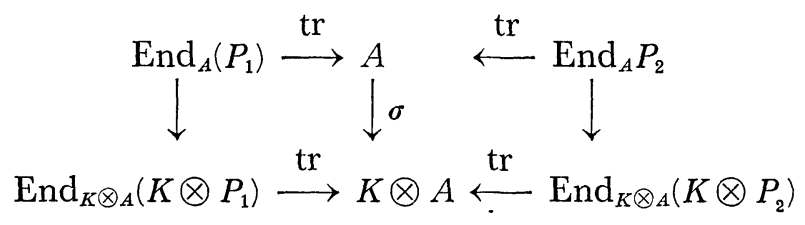

as in (1.4). Since $A$ is $\sigma$-torsion free, $\sigma$ is a monomorphism. Now $K \otimes P_{1} \cong K \otimes P_{2}$ implies $r_{K \otimes A}\left(K \otimes P_{1}\right)=r_{K} \otimes_{A}\left(K \otimes P_{2}\right)$. By commutativity of the diagram, and injectivity of $\sigma, r_{A}\left(P_{1}\right)=r_{A}\left(P_{2}\right)$ follows. Now apply (4.1) to deduce $P_{1} \cong P_{2}$. The converse is clear.

\section{REFFERENCES}

[1] C.W. Curtis, I. Reiner, Representation Theory of Finite Groups and Associative Algebras. Interscience Publ., New York (1962).

[2] A. Grothendieck, Elements de Geometrie Algebrique, Institute des Hautes Etudes Scientifique, Publ. Math, No. 4 (1960).

[ 3 ] A. Hattori, Rank Element of a Projective Module, Nagoya Math J. 25(1965), 113-120.

[4] M. Nagata, Local Rings, Interscience Publ., New York (1962).

[5] H. Röhrl, A Categorical Setting for Traces and Determinants, to appear Nagoya Math J., 34 (1968).

[6] O. Zariski, P. Samuel, Commutative Algebra vol. II, D. Van Nostrand Co., Inc., Princeton, 1960. 